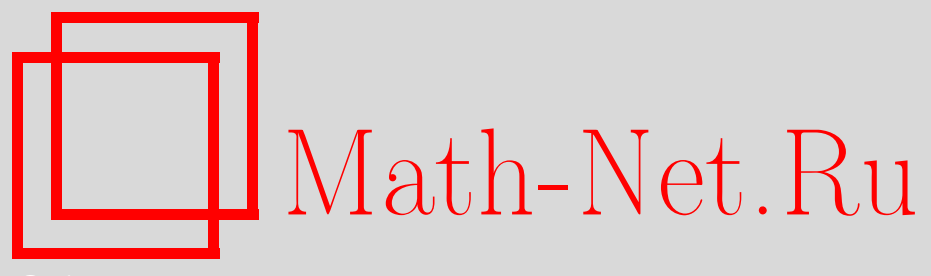

В. С. Владимиров, О нелинейных уравнениях $p$ адических открытых, замкнутых и открыто-замкнутых струн, ТMФ, 2006, том 149, номер 3, 354-367

DOI: https://doi.org/10.4213/tmf5522

Использование Общероссийского математического портала Math-Net.Ru подразумевает, что вы прочитали и согласны с пользовательским соглашением http://www . mathnet.ru/rus/agreement

Параметры загрузки:

IP : 35.173 .219 .149

26 апреля 2023 г., 17:12:26

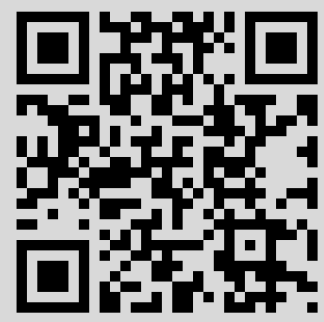




\section{О НЕЛИНЕЙНЫХ УРАВНЕНИЯХ p-АДИЧЕСКИХ ОТКРЫТЫХ, ЗАМКНУТЫХ И ОТКРЫТО-ЗАМКНУТЫХ СТРУН}

Исследуется структура решений краевых задач для одномерной нелинейной системы псевдодифференциальных уравнений, описывающей динамику (роллинг) $p$-адической открытой, замкнутой и открыто-замкнутой струн для скалярного поля тахионов. Применяется метод последовательных приближений. Для открыто-замкнутой струны доказана его сходимость при нечетных $p$ вида $p=4 n+1$ при условии, что известно решение для замкнутой струны. При $p=2$ обсуждаются вопросы существования и несуществования решения краевых задач и указывается на возможность появления разрывных решений.

Ключевые слова: струны, тахионы.

K 80-летию Анатолия Алексеевича Логунова

\section{1. ВВЕДЕНИЕ}

Для описания динамики тахионов открытой и замкнутой $p$-адических струн был предложен лагранжиан [1]

$$
\begin{aligned}
\mathfrak{L}=\frac{1}{h^{2}} & {\left[-\frac{p^{4}}{2\left(p^{2}-1\right)} \Psi p^{-\square / 4} \Psi+\frac{p^{4}}{p^{4}-1} \Psi^{p^{2}+1}\right]+} \\
& +\frac{1}{g^{2}}\left[-\frac{p^{2}}{2(p-1)} \Phi p^{-\square / 2} \Phi+\frac{p^{2}}{p^{2}-1} \Psi^{p(p-1) / 2}\left(\Phi^{p+1}-1\right)\right],
\end{aligned}
$$

где $\Psi(t, x)$ и $\Phi(t, x), x=\left(x_{1}, x_{2}, \ldots, x_{d-1}\right)$, - тахионные поля для открытых и замкнутых струн, $h$ и $g$ - константы взаимодействия между открытыми и замкнутыми струнными секторами, $\square=-\partial_{t}^{2}+\nabla_{x}^{2}-d$-мерный даламбертиан, $p-$ простое число, $p=2,3,5, \ldots$ (впредь $p$ будем считать целым числом, бо́льшим 1$)$.

* Математический институт им. В. А. Стеклова РАН, Москва, Россия. E-mail: vladim@mi.ras.ru 
Соответствующие уравнения движения, вытекающие из (1.1) при $\lambda^{2}=h^{2} / g^{2} \neq 0$, имеют вид [2]

$$
\begin{gathered}
\Psi^{p^{2}}-p^{-\square / 4} \Psi+\lambda^{2} \frac{p-1}{2 p} \Psi^{p(p-1) / 2-1}\left(\Phi^{p+1}-1\right)=0, \\
\Phi^{p} \Psi^{p(p-1) / 2}-p^{-\square / 2} \Phi=0 .
\end{gathered}
$$

Совершив в системе уравнений (1.2) предельный переход $\lambda^{2} \rightarrow 0$, получим следующую упрощенную систему уравнений для открыто-замкнутой струны [2]:

$$
\begin{aligned}
\Psi^{p^{2}} & =p^{-\square / 4} \Psi, \\
\Phi^{p} \Psi^{p(p-1) / 2} & =p^{-\square / 2} \Phi .
\end{aligned}
$$

Система уравнений (1.3) включает уравнение (1.3а), описывающее динамику поля $\Psi$ замкнутой струны, и уравнение (1.3б), описывающее динамику поля $\Phi$ открытой струны при известном поле $\Psi$.

При $\Psi=1$ система (1.3) превращается в уравнение для открытой струны

$$
\Phi^{p}=p^{-\square / 2} \Phi
$$

Уравнения (1.3) имеют следующие вакуумные решения:

$$
\begin{array}{ll}
(\Psi=0, \Phi=0) \quad \forall p ; & (\Psi=1, \quad \Phi=1), \quad p \text { четное; } \\
(\Psi=1, \Phi= \pm 1), p=4 n+3 ; & (\Psi= \pm 1, \Phi= \pm 1), p=4 n+1 .
\end{array}
$$

При $d=1$ система (1.3) и отдельно уравнения (1.3а) и (1.4) описывают движение (роллинг) тахионов по времени для открыто-замкнутой, замкнутой и открытой струн, соответственно. В этом случае уравнения (1.3) принимают вид

$$
\begin{aligned}
\Psi^{p^{2}} & =p^{\partial_{t}^{2} / 4} \Psi, \\
\Phi^{p} \Psi^{p(p-1) / 2} & =p^{\partial_{t}^{2} / 2} \Phi .
\end{aligned}
$$

Сделаем замену аргументов у полей:

$$
\psi(t)=\Psi(t \sqrt{2 \ln p}), \quad \varphi(t)=\Phi(t \sqrt{2 \ln p}) .
$$

В классе измеримых функций $(\psi, \varphi)$, удовлетворяющих условию роста (5.2) (см. ниже) при $\gamma=1$ или $\gamma=2$ уравнения (1.6) превращаются в классическую систему нелинейных интегральных уравнений

$$
\begin{aligned}
\psi^{p^{2}}(t) & =\sqrt{\frac{2}{\pi}} \int_{-\infty}^{\infty} e^{-2(t-\tau)^{2}} \psi(\tau) d \tau, \quad t \in \mathbb{R}, \\
\varphi^{p}(t) \psi^{p(p-1) / 2}(t) & =\frac{1}{\sqrt{\pi}} \int_{-\infty}^{\infty} e^{-(t-\tau)^{2}} \varphi(\tau) d \tau, \quad t \in \mathbb{R} .
\end{aligned}
$$

Аналогичный вид принимает и уравнение (1.4) (уравнение (1.8б) при $\psi=1$ ) для открытой струны:

$$
\varphi^{p}(t)=\frac{1}{\sqrt{\pi}} \int_{-\infty}^{\infty} e^{-(t-\tau)^{2}} \varphi(\tau) d \tau, \quad t \in \mathbb{R} .
$$


В соответствии с вакуумными решениями (1.5) ставятся следующие краевые условия: для уравнения (1.8а)

$$
\lim _{t \rightarrow-\infty} \psi(t)=\lim _{t \rightarrow \infty} \psi(t)=1
$$

для уравнений (1.8б) и (1.9)

$$
\lim _{t \rightarrow \infty} \varphi(t)=1, \quad \lim _{t \rightarrow-\infty} \varphi(t)=\left\{\begin{array}{lll}
-1, & p & \text { нечетное } \\
0, & p & \text { четное }
\end{array}\right.
$$

Система уравнений (1.3) является нелинейной с псевдодифференциальными членами с символами $p^{-\xi^{2} / 4}, p^{-\xi^{2} / 2}, \xi^{2}=t^{2}-x_{1}^{2}-\cdots-x_{d-1}^{2}$. Поэтому их естественно рассматривать в некоторых алгебрах обобщенных функций из $\mathfrak{D}^{\prime}\left(\mathbb{R}^{d}\right)$, преобразования Фурье которых являются аналитическими функционалами из пространства $Z^{\prime}$ [3], [4]. Физический интерес представляют только вещественные решения этих уравнений, впредь будут рассматриваться только такие решения.

Если $(\Psi(t, x), \Phi(t, x))$ есть решение системы (1.3), то все его сдвиги и все его отражения также являются ее решениями. В случае нечетного $p$ вида $p=4 n+$ $1(-\Psi(t, x),-\Phi(t, x))$ тоже является решением этой системы. Поэтому решение системы (1.3) не единственно (если оно существует).

Исследованию этого нового класса уравнений с бесконечным числом производных посвящены многие работы физиков и математиков с широким использованием компьютерной техники (см. [1], [2], [4]-[14] и приведенную там литературу). В струнной теории поля взаимодействие нелокально [5], что существенно отличает ее от классической локальной теории поля. Эти уравнения представляют значительный интерес не только для $p$-адической математической физики, но и для космологии [7], [9], [13]. По существу, эти задачи относятся к классическому математическому анализу: от $p$-адических чисел здесь осталось только целое число $p$, к тому же оно не обязано быть простым.

В этой работе в рамках предложенной модели изучается структура решений для открытых, замкнутых и открыто-замкнутых струн. В разделе 2 перечислены известные математические результаты для открытой струны (краевая задача (1.9), (1.11)). В разделе 3 многие результаты для открытой струны почти без изменений переносятся и на замкнутую струну (краевая задача (1.8а), (1.10)). При нечетном $p$ применен метод последовательных приближений для четных решений с двумя нулями. При четном $p$ доказано, что не существует непрерывных четных неубывающих при $t>0$ нетривиальных решений (теорема 1) (см. также [8]). Поэтому в этом случае непрерывные решения должны иметь либо не меньше четырех нулей, либо быть разрывными со скачками первого рода. В разделе 4 для открыто-замкнутой струны (краевая задача $(1.8),(1.10),(1.11))$ при $p$ вида $p=4 n+1$ доказывается сходимость метода последовательных приближений для краевой задачи (1.8б), (1.11) для нечетного решения с одним нулем при условии, что известно четное решение краевой задачи (1.8а), (1.10) (теорема 2). Дано описание структуры решений. В разделе 5 приведены необходимые свойства интегрального оператора $K_{\gamma}=e^{1 /(4 \gamma) \partial_{t}^{2}}$. 


\section{2. ОТКРЫТАЯ СТРУНА}

Динамика открытой струны определяется интегральным уравнением (1.9) и граничными условиями (1.11).

О решениях $\varphi$ уравнения (1.9) справедливы следующие утверждения.

УтВеРЖДЕНИЕ 1. Если решение $\varphi(t)$ ограничено, то функиии $\varphi(t)=0, \pm 1$ при нечетном $p$ и $\varphi(t)=0,1$ при четном $p$ являются решениями; если решение $\varphi(t)$ не сводится $к$ постоянной, то оно кусочно-аналитическое (при нечетном р непрерывное) с оценкой

$$
|\varphi(t)|<1, \quad t \in \mathbb{R} .
$$

УтверЖДЕНИЕ 2. Если решение $\varphi(t) \rightarrow$ а при $t \rightarrow \infty,|a|<\infty$, то $a=0$ или $a= \pm 1$ при нечетном $p ; a=0$ или $a=1$ при четном $p$. При этом $\left(\varphi^{p}\right)^{\prime}(t) \rightarrow 0$ при $t \rightarrow \infty$, а если $a \neq 0$, то $\varphi^{\prime}(t) \rightarrow 0$ при $t \rightarrow \infty$.

УтВЕРЖДЕНИЕ 3. Интегральное уравнение (1.9) эквивалентно следующей краевой задаче для уравнения теплопроводности [11]:

$$
\begin{array}{rlrl}
u_{x} & =\frac{1}{4} u_{t t}, & & 0<x \leqslant 1, \quad t \in \mathbb{R}, \\
u(0, t) & =\varphi(t), \quad u(1, t)=\varphi^{p}(t), \quad t \in \mathbb{R} .
\end{array}
$$

Обратим внимание, что переменные $x$ и $t$ в уравнении (2.2) поменялись местами по сравнению с классическим уравнением теплопроводности.

Решением краевой задачи $(2.2),(2.3)$ назовем всякую измеримую функцию $u(x, t)$, ограниченную условием роста (5.2) по $t$ при $\gamma=1$. Функция $u(x, t)$ называется интерполирующей между решением $\varphi$ и его степенью $\varphi^{p}$.

Отметим, что интерполирующая функция представляется формулой Пуассона для уравнения (2.2):

$$
u(x, t)=\frac{1}{\sqrt{\pi x}} \int_{-\infty}^{\infty} \varphi(\tau) e^{-(t-\tau)^{2} / x} d \tau, \quad 0<x \leqslant 1 .
$$

Для нулей интерполирующей функции $u(x, t)$ имеет место следующее утверждение.

УтВеРЖДЕНИЕ 4 (теорема о ветвлении нулей функции $u(1, t)=\varphi^{p}(t)$ ) [11], [14]. Пусть функиия $u(x, t)$ имеет в точке $t=0$ нуль четной кратности $2 n$. Тогда уравнение

$$
u(1-\varepsilon, t)=0 \quad \text { npu } \quad \varepsilon \rightarrow+0
$$

имеет ровно 2 р различных простых вещественных нулей:

$$
t_{k}^{ \pm}(\varepsilon)= \pm \lambda_{k} \sqrt{\varepsilon}+O(\varepsilon), \quad k=1,2, \ldots, n,
$$

где $\lambda_{k}, k=1,2, \ldots, n,-$ положительные корни полинома Эрмита: $H_{2 n}(\lambda)=0$.

Например, при $n=2$ имеем $\lambda^{4}-12 \lambda^{2}+12=0$, так что

$$
\lambda_{1}=\sqrt{6-2 \sqrt{6}} \approx 1.049, \quad \lambda_{2}=\sqrt{6+2 \sqrt{6}} \approx 3.301 .
$$


УтВЕРЖДЕНИЕ 5. Если решение $\varphi \in \mathfrak{L}_{2}^{1}$, то оно разлагается в ряд по полиномам Эрмита [11]

$$
\varphi(t)=\sum_{n=0}^{\infty} a_{n} \frac{H_{n}(t)}{2^{n} n !}, \quad a_{n}=\left(\varphi, H_{n}\right)_{1},
$$

а функция $\varphi^{p}(t)-$ в ряд Тейлора

$$
\varphi^{p}(t)=\sum_{n=0}^{\infty} a_{n} \frac{t^{n}}{n !}
$$

сходящийся равномерно на каждом компакте из $\mathbb{R}$. Если же $\varphi \in \mathfrak{L}_{2}^{1 / 2}$, то справедливы равенства

$$
\left(\varphi^{p}, H_{n}\right)_{1}=\left(\varphi, V_{n}\right)_{1 / 2}, \quad n=0,1, \ldots,
$$

где $V_{n}-$ модифицированнье полиномь Эрмита,

$$
V_{n}(t)=2^{-n / 2} H_{n}\left(\frac{t}{\sqrt{2}}\right), \quad n=0,1, \ldots .
$$

Определение пространства $\mathfrak{L}_{2}^{\alpha}$ дано в разделе 5 .

УтвеРЖДЕНИЕ 6. Пусть $\varphi(t)$ - решение уравнения (1.9) и $t=0$ - нуль функиии $\varphi^{p}(t)$ кратности $\sigma \geqslant 1$. Тогда справедливы соотношения [4]

$$
\frac{2^{n}}{\sqrt{\pi}} \int_{-\infty}^{\infty} \varphi(\tau) \tau^{n} e^{-\tau^{2}} d \tau= \begin{cases}0, & n=0,1, \ldots, \sigma-1 \\ a \neq 0, & n=\sigma .\end{cases}
$$

Число перемен знака у функиии $\varphi(t)$ не менъше $\sigma$ [11].

УтвЕРЖДЕНИЕ 7а. При нечетных $р$ и $\sigma$ решение $\varphi(t)$ меняет знак в нуле $и$

$$
\varphi(t)=\left(\frac{a}{\sigma !}\right)^{1 / p} t^{\sigma / p}[1+O(t)] \quad \text { npu } \quad t \rightarrow 0 .
$$

УТВЕРЖДЕНИЕ 7б. При четном $р$ кратность $\sigma$ четна и $а>>0$, и если $\varphi(t)$ меняет знак в нуле, то

$$
\varphi(t)=\left(\frac{a}{\sigma !}\right)^{1 / p} \operatorname{sign} t|t|^{\sigma / p}[1+O(t)] \quad \text { npu } \quad t \rightarrow 0 .
$$

УТВЕРЖДЕНИЕ 7в. Если $\varphi(t)$ не меняет знака в нуле, то а $>0$ и кратность $\sigma$ четна, $и$

$$
\varphi(t)=\left(\frac{a}{\sigma !}\right)^{1 / p}|t|^{\sigma / p}[1+O(t)] \quad \text { npu } \quad t \rightarrow 0 .
$$

О решениях краевой задачи (1.9), (1.11) справедливы следующие утверждения.

УТВеРЖДЕНИЕ 8. Для нечетного р существует непрерывное, вещественно-аналитическое при $t \neq 0$, нечетное, возрастающее решение $\varphi(t)$ с одним простым нулем $t=0$, при этом справедливы соотношения (2.11) и (2.12) при $\sigma=1$ [4], [10], [12]. 
УтВеРЖДЕНИЕ 9. Для четного р не существует непрерывных решений, которые либо неотрицательны, либо имеют одну перемену знака; если существует непрерывное решение с двумя нулями, то оно имеет ровно две перемены знака [11]. Возможны разрывные решения с разрывами первого рода.

УтВеРЖДЕНИЕ 10. Пусть $u(x, t)$ - интерполирующая функиия между решением $\varphi$ и его степенъю $\varphi^{p}$. Тогда справедливы закон сохранения

$$
\int_{-\infty}^{\infty}[\varphi(t)-u(x, t)] d t=0=\int_{-\infty}^{\infty}\left[\varphi(t)-\varphi^{p}(t)\right] d t, \quad x \geqslant 0,
$$

и неравенство

$$
\left|\int_{-\infty}^{a}[u(x, t)-\varphi(t)] d t\right|<\sqrt{\frac{x}{\pi}}, \quad x>0, \quad a \in \mathbb{R} .
$$

УтВеРЖДЕНИЕ 11. Для нечетного р справедливы включения

$$
1-\varphi^{p-1}, 1-|u(x, \cdot)| \in \mathfrak{L}_{1}(\mathbb{R}), \quad x \geqslant 0,
$$

число нулей функции $\varphi^{p}(t)$ конечно, все ее нули имеют конечную кратность; число перемен знака решения $\varphi(t)$ не больше числа нулей $\varphi^{p}(t)$ и не меньше максимальной кратности нулей этой функиии; если $\varphi^{p}(t)$ имеет толъко три нуля, то решение $\varphi(t)$ имеет три перемены знака [11].

УТВЕРЖДЕНИЕ 12. Для четного р справедливы включения

$$
1-\varphi^{p-1}, 1-u(x, \cdot) \in \mathfrak{L}_{1}(0, \infty), \quad x \geqslant 0,
$$

и сходится интеграл

$$
\int_{-\infty}^{0} \varphi(t)\left[1-\varphi^{p-1}(t)\right] d t
$$

а если функция $\varphi(t)$ знакопостоянна при $t<c$, то справедливы включения

$$
\varphi, u(x, \cdot) \in \mathfrak{L}_{1}(-\infty, 0), \quad x \geqslant 0 .
$$

Множество нулей функции $\varphi^{p}(t)$ конечно или счетно и ограничено сверху. Обозначим эти нули через $t_{0}, t_{1}, \ldots, t_{k} \rightarrow-\infty$, а их кратности - через $\sigma_{0}, \sigma_{1}, \ldots$ [11]. По теореме Адамара (лемма 2 раздела 5) справедливо неравенство

$$
\sum_{k=0}^{\infty} \sigma_{k}\left|t_{k}\right|^{-2-\varepsilon}<\infty \quad \text { при любом } \varepsilon>0 .
$$

Возникают следующие вопросы.

1. Существует ли непрерывное или разрывное решение краевой задачи (1.9), (1.11) при четном $p$ ?

2. Всегда ли в нуле функции $\varphi^{p}(t)$ имеет место перемена знака у решения $\varphi(t)$ ?

3. Являются ли кратности нулей функции $\varphi^{p}(t)$ только нечетными при нечетном $p$ и только четными вида $2(2 n+1)$ при четном $p$ ? 


\section{3. ЗАМКНУТАЯ СТРУНА}

Динамика замкнутой струны определяется интегральным уравнением (1.8а) и краевыми условиями (1.10). Уравнение (1.8а) сводится к уравнению (1.9) с заменой $p$ на $p^{2}$ и оператора $K_{1}$ на оператор $K_{2}$ (см. раздел 5$)$. Поэтому утверждения $1-6$ раздела 2 , касающиеся ограниченных решений уравнения (1.9), остаются справедливыми и для уравнения (1.8а). Что касается краевой задачи (1.8a), (1.10), то утверждения 9-11 раздела 2 остаются справедливыми, а утверждения 8 и 12 заменяются на следующие.

УтвеРЖДЕНИЕ 8'. Не существует неотрицательных непрерывных решений, кроме $\psi(t)=1$. При четном р возможны разрывные решения со разрывами первого рода [4].

УтВеРЖДЕНИЕ 12'. Число нулей функиии $\psi^{p^{2}}$ конечно как при нечетном, так и при четном $p$, и поэтому справедливы включения

$$
1-\psi^{p^{2}-1}, 1-u(x, \cdot) \in \mathfrak{L}_{1}(\mathbb{R}), \quad x \geqslant 0 .
$$

Для построения приближенного решения краевой задачи (1.8а), (1.10) воспользуемся методом последовательных приближений, разработанным в работах [10] и [4] для открытой струны при нечетных $p$. Будем искать четное решение $\psi(t)$ с двумя нулями $\pm t_{0}$. Последовательные приближения определим рекуррентной формулой:

$$
\psi_{n+1}(t)=\left[\left(K_{2} \psi_{n}\right)(t)\right]^{1 / p^{2}}, \quad n=0,1, \ldots, \quad \psi_{0}(t)=1-\beta e^{-\alpha t^{2}} .
$$

Здесь оператор $K_{2}$ определен формулой (5.1) (см. ниже) при $\gamma=2$. На четных функциях $\psi$ он принимает вид

$$
\left(K_{2} \psi\right)(t)=\sqrt{\frac{2}{\pi}} \int_{0}^{\infty} \psi(\tau)\left[e^{-2(t-\tau)^{2}}+e^{-2(t+\tau)^{2}}\right] d \tau .
$$

Вычислим второе приближение. Имеем

$$
\left(K_{2} \psi_{0}\right)(t)=\sqrt{\frac{2}{\pi}} \int_{-\infty}^{\infty}\left[1-\beta e^{-\alpha \tau^{2}-2(t-\tau)^{2}}\right] d \tau=1-\beta \sqrt{\frac{2}{\alpha+2}} e^{-2 \alpha t^{2} /(\alpha+2)},
$$

откуда в силу (3.2) выводим

$$
\psi_{1}(t)=\left[1-\beta \sqrt{\frac{2}{\alpha+2}} e^{-2 \alpha t^{2} /(\alpha+2)}\right]^{1 / p^{2}} .
$$

Наконец,

$$
\psi_{2}(t)=\left\{\sqrt{\frac{2}{\pi}} \int_{0}^{\infty}\left[1-\beta \sqrt{\frac{2}{\alpha+2}} e^{-2 \alpha \tau^{2} /(\alpha+2)}\right]^{1 / p^{2}}\left[e^{-2(t-\tau)^{2}}+e^{-2(t+\tau)^{2}}\right] d \tau\right\}^{1 / p^{2}} .
$$

Построенная по формуле (3.3) функция $\psi_{2}(t)$, как и функция $\psi_{1}(t),-$ хорошее приближение для решения краевой задачи (1.8a),(1.10). Для того, чтобы найти нули $\pm t_{0}$ функции $\psi_{2}^{p^{2}}(t)$ необходимо решить уравнение

$$
\int_{0}^{\infty}\left[1-\beta \sqrt{\frac{2}{\alpha+2}} e^{-2 \alpha \tau^{2} /(\alpha+2)}\right]^{1 / p^{2}}\left[e^{-2(t-\tau)^{2}}+e^{-2(t+\tau)^{2}}\right] d \tau=0 .
$$


ПримеРЫ. Пусть $p=3, \alpha=0,1$. Тогда:

1) при $\beta=1.5, \psi_{0}(0)=-0.500, t_{0}^{0}=2.01, \psi_{1}(0)=-0.917, t_{0}^{1}=2.00$;

2) при $\beta=1.8, \psi_{0}(0)=-0.800, t_{0}^{0}=2.42, \psi_{1}(0)=0.971, t_{0}^{1}=2.43$;

3) при $\beta=1.9, \psi_{0}(0)=-0.900, t_{0}^{0}=2.53, \psi_{1}(0)=-0.982, t_{0}^{1}=2.55$;

4) при $\beta=2, \psi_{0}(0)=-1, t_{0}^{0}=2.63, \psi_{1}(0)=-0.995, t_{0}^{1}=2.65$.

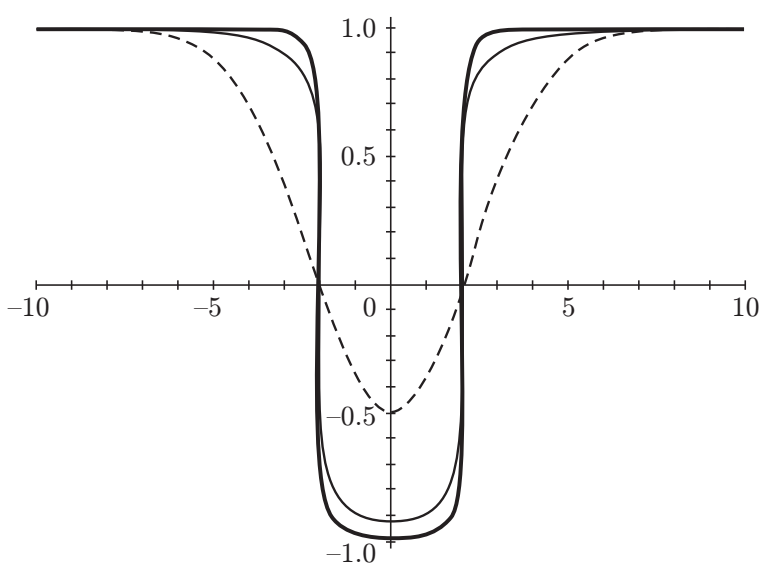

Рис. 1

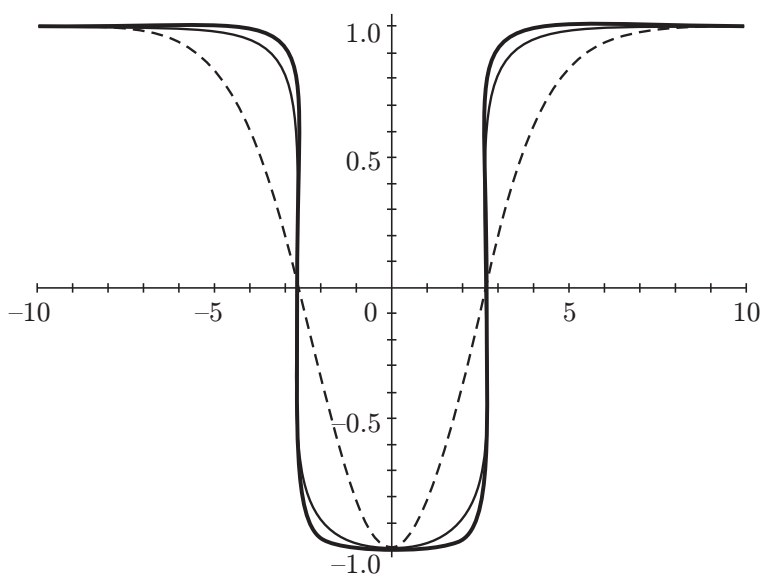

Рис. 2

На рис. 1 ( $\left.\beta=1.5, t_{0}^{n} \approx 2.00\right)$ и рис. $2\left(\beta=2, t_{0}^{n} \approx 2.65\right)$ изображены итерации $\psi_{n+1}^{9}=\left(K_{2} \psi_{n}\right)^{1 / 9}: \psi_{0}$ - штриховая линия, $\psi_{1}$ - тонкая сплошная линия, $\psi_{2}-$ жирная линия.

Как показывают численные расчеты (см. примеры и рисунки), при каждом $\beta$ из интервала $1.5 \leqslant \beta \leqslant 2$ нулевое приближение $\psi_{0}=1-e^{-0.1 t^{2}}$ определяет решение, зависящее от $\beta$, к которому быстро сходятся итерации (и поэтому нули $t_{0}^{n}, n=0,1,2$, 
на рис. 1, 2 неразличимы). Таким образом, мы имеем однопараметрическое семейство приближенных решений, зависящих от параметра $\beta$ в начальной функции $\psi_{0}$. Однако доказательство сходимости последовательных приближений, продемонстрированное в работах [4], [10], [12] для открытой струны, в данном случае не проходит. Тем не менее справедливо

УтВеРЖДЕНИЕ 13. Пусть р нечетное. Если существует непрерывное четное решение $\psi(t)$ с двумя нулями $t= \pm t_{0}$ краевой задачи (1.8а), (1.10), то эти нули простые и справедливы соотношения

$$
\psi(t)=\left[a\left(t \pm t_{0}\right)\right]^{p^{-2}}\left[1+O\left(\left|t \pm t_{0}\right|\right)\right], \quad t \rightarrow \pm t_{0},
$$

где

$$
a=\sqrt{\frac{2}{\pi}} \int_{0}^{\infty} \psi(\tau)\left[e^{-2\left(t_{0}-\tau\right)^{2}}+e^{-2\left(t_{0}+\tau\right)^{2}}\right] d \tau .
$$

ДоказАТЕльство. Убедимся, что нули $\pm t_{0}$ простые. Действительно, если нуль $t_{0}$ кратный, то необходимо, чтобы первая производная функции $\psi^{p^{2}}(t)$ обращалась в нуль в точке $t_{0}$. Но $\psi(t)$, а значит, и $\psi^{p^{2}}(t)$ меняют знаки в точке $t_{0}$. Стало быть, и вторая производная функции $\psi^{p^{2}}(t)$ равна нулю в этой точке. Это значит, что кратность нуля $t_{0}$ этой функции не меньше 3 , но тогда и число перемен знака ее должно быть не меньше 3 (см. утверждение 6), что противоречит нашему предположению.

Для краевой задачи (1.8а), (1.10) при четных $p$ справедлив такой отрицательный результат, сформулированный впервые в работе [8].

Теорема 1. Не существует непрерывных четных неубывающих при $t>0$ решений $\psi$, кроме $\psi \equiv 1$.

ДокАзАтЕЛьство. Пусть $\psi(t) \not \equiv 1$ - непрерывное четное и неубывающее при $t>0$ решение краевой задачи $(1.8 \mathrm{a}),(1.10)$. Тогда $\psi(0)<0$, иначе в силу наших предположений функция $\psi(t)$ была бы неотрицательной при всех $t \in \mathbb{R}$, что противоречит утверждению $8^{\prime}$. Поэтому существует такая точка $t=t_{0}$, что $\psi(t)<0$ при всех $0 \leqslant t<t_{0}$ и $\psi\left(t_{0}\right)=0$. С другой стороны, функция $\psi^{\prime}(t), t \geqslant 0$, неотрицательна и непрерывна везде, кроме точки $t_{0}$, в которой она имеет интегрируемую особенность (см. утверждения 7). Кроме того, $\psi^{\prime}(\infty)=0$ (см. утверждение 2). Согласно лемме 4 (см. раздел 5) справедливо равенство

$$
p^{2} \psi^{p^{2}-1}(t) \psi^{\prime}(t)=\sqrt{\frac{2}{\pi}} \int_{0}^{\infty} \psi^{\prime}(\tau)\left[e^{-2(t-\tau)^{2}}-e^{-2(t+\tau)^{2}}\right] d \tau \geqslant 0,
$$

которое приводит к противоречию при $0<t<t_{0}$, поскольку число $p^{2}-1$ нечетное и потому $\psi^{p^{2}-1}(t)<0$ при $0<t<t_{0}$.

Смысл доказанного утверждения состоит в том, что нетривиальные четные решения краевой задачи (1.8а), (1.10) должны иметь либо четное число нулей, не меньшее четырех (см. утверждение 11), либо они разрывны. 


\section{4. ОТКРЫТО-ЗАМКНУТАЯ СТРУНА}

Динамика открыто-замкнутой струны определяется системой уравнений (1.8) и краевыми условиями (1.10) и (1.11). Краевая задача (1.8а), (1.10) описывает замкнутую струну, и решение этой задачи обсуждалось в разделе 3 . Пусть $\psi_{0}(t)$ - известное четное решение этой задачи такое, что кратности $\sigma_{k}, k=1,2, \ldots, m$, нулей функции $\psi_{0}^{p^{2}}(t)$ удовлетворяют неравенству

$$
\sigma_{k}<\frac{2 p^{2}}{p-1}, \quad k=1,2, \ldots, m .
$$

Подставляя это решение в уравнение (1.8б), получим уравнение

$$
\varphi^{p}(t) \psi_{0}^{p(p-1) / 2}(t)=\left(K_{1} \varphi\right)(t),
$$

где оператор $K_{1}$ определен формулой (5.1) при $\gamma=1$. Для решения уравнения (4.2) введем новую неизвестную функцию

$$
\chi(t)=\varphi(t) \psi_{0}^{(p-1) / 2}(t), \quad \varphi(t)=\chi(t) \psi_{0}^{-(p-1) / 2}(t) .
$$

Подставляя ее в уравнение (4.2), получим интегральное уравнение

$$
\chi^{p}(t)=\left(K_{1} v \chi\right)(t)
$$

где введено обозначение

$$
v(t)=\psi_{0}^{-(p-1) / 2}(t) .
$$

Перечислим свойства функции $v:|v(t)|>1, t \in \mathbb{R}$, эта функция четная, (вещественно)-аналитическая всюду, кроме конечного числа нулей функции $\psi_{0}(t)$, в которых $v$ в силу условия (4.1) имеет интегрируемую особенность (см. утверждения 7а, 7в с заменой $p$ на $\left.p^{2}\right)$, удовлетворяет краевым условиям $(1.10)$, и в силу (3.1) (поскольку $\left.p^{2}-1>(p-1) / 2\right)$ справедливы включения

$$
v-1,|v|-1 \in \mathfrak{L}_{1}(\mathbb{R}) .
$$

Докажем оценку

$$
\left(K_{1}|v|\right)(t)<N, \quad t \in \mathbb{R}, \quad N=1+\frac{2}{\sqrt{\pi}} \int_{0}^{\infty}[|v(\tau)|-1] d \tau .
$$

Действительно, в силу (4.6) имеем

$$
\left(K_{1}|v|\right)(t)<\frac{1}{\sqrt{\pi}} \int_{-\infty}^{\infty}[|v(\tau)|-1] e^{-(t-\tau)^{2}} d \tau+1 .
$$

Из оценки (4.7) следует оценка

$$
|\chi(t)|<N^{1 /(p-1)}, \quad t \in \mathbb{R} .
$$

В самом деле, из уравнения (4.4) имеем

$$
\left|\chi^{p}(t)\right|=|\chi(t)|^{p}=|K(v \chi)(t)|<\max _{t \in \mathbb{R}}|\chi(t)|(K|v|)(t)<N \max _{t \in \mathbb{R}}|\chi(t)|,
$$


откуда и следует оценка (4.8).

Из (4.3) и (4.8) вытекает оценка для решения:

$$
|\varphi(t)|<N^{1 /(p-1)}\left|\psi_{0}^{-(p-1) / 2}(t)\right|, \quad t \in \mathbb{R} .
$$

Предположим теперь, что $p-$ нечетное число вида $p=4 n+1$. В этом случае в силу (4.5) $v(t)>1, t \in \mathbb{R}$. Как и для открытой струны [4], [10], ищем нечетное решение $\chi(t)$ краевой задачи (4.4), (1.11) методом последовательных приближений:

$$
\chi_{n+1}(t)=\left[\left(K_{1} v \chi_{n}\right)(t)\right]^{1 / p}, \quad n=0,1, \ldots, \quad \chi_{0}(t)=\operatorname{sign} t, \quad t \in \mathbb{R} .
$$

Приближения $\chi_{n}(t), n=1,2, \ldots,-$ нечетные, непрерывные, положительные, возрастающие при $t>0$ функции, они обращаются в нуль при $t=0$ и стремятся к единице при $t \rightarrow \infty$. Целые функции $\chi_{n}^{p}(t), n=1,2, \ldots$, имеют простой нуль при $t=0$. Простота нуля вытекает из (4.10) в силу

$$
\frac{d}{d t} \chi_{n}^{p}(0)=\frac{d}{d t}\left(K_{1} v \chi_{n-1}\right)(0)=\frac{4}{\sqrt{\pi}} \int_{0}^{\infty} v(\tau) \chi_{n-1}(\tau) \tau e^{-\tau^{2}} d \tau>0 .
$$

Докажем, что существуют такие положительные числа $\eta$ и $\theta$, что

$$
\eta \chi_{1}(t) \leqslant \chi_{2}(t) \leqslant \theta \chi_{1}(t), \quad t \geqslant 0 .
$$

Введем функцию $f(t)=\chi_{2}^{p}(t) \chi_{1}^{-p}(t)$. Она непрерывна и положительна при $t>0$ и обращается в единицу при $t \rightarrow \infty$; ее предел при $t \rightarrow 0$ в силу (4.11) существует и конечен (по правилу Лопиталя). Следовательно, существуют такие числа $a$ и $b$, $0<a<b$, что

$$
a \leqslant f(t) \leqslant b, \quad t \geqslant 0,
$$

откуда и вытекает неравенство (4.12) при $\eta=a^{1 / p}$ и $\sigma=b^{1 / p}$.

Умножая неравенства (4.12) на $v$ и применяя оператор $K_{1}$, с учетом неотрицательности ядра оператора $K_{1}$ получим неравенства

$$
\eta\left(K_{1} v \chi_{1}\right)(t) \leqslant\left(K_{1} v \chi_{2}\right)(t) \leqslant \theta\left(K_{1} v \chi_{1}\right)(t),
$$

откуда, пользуясь (4.10), выводим

$$
\eta \chi_{2}^{p}(t) \leqslant \chi_{3}^{p}(t) \leqslant \theta \chi_{2}^{p}(t)
$$

и, следовательно,

$$
\eta^{1 / p} \chi_{2}(t) \leqslant \chi_{3}(t) \leqslant \theta^{1 / p} \chi_{2}(t) .
$$

И так далее. В результате получим следующие неравенства:

$$
\eta^{p^{-n+1}} \chi_{n}(t) \leqslant \chi_{n+1}(t) \leqslant \theta^{p^{-n+1}} \chi_{n}(t), \quad n=2,3, \ldots, \quad t \geqslant 0 .
$$

Теперь, рассуждая как в работе [4], убеждаемся, что последовательность итераций $\chi_{n}(t), n=0,1, \ldots$, сходится равномерно на $\mathbb{R}$ к решению $\chi(t)$ краевой задачи $(4.3),(1.11)$ при $p$ вида $p=4 n+1$. Итак, мы доказали следующую теорему. 
Теорема 2. Пусть $p \equiv 1(\bmod 4)$ и $\psi_{0}$ - четное решение краевой задачи $(1.8 \mathrm{a})$, $(1.10)$, причем кратности нулей суть $\sigma_{k}, k=1,2, \ldots, m, \oint у н к ц и и ~ \psi_{0}^{p^{2}}(t)$ удовлетворяют условию (4.1). Тогда решение краевой задачи (1.8), (1.10), (1.11) для открытозамкнутой струны существует и задается формулой

$$
\left(\psi=\psi_{0}, \quad \varphi=\chi \psi_{0}^{-(p-1) / 2}\right)
$$

с оценкой

$$
|\varphi(t)| \leqslant C \psi_{0}^{-(p-1) / 2}(t), \quad t \in \mathbb{R} ; \quad 1-|\varphi| \in \mathfrak{L}_{1}(\mathbb{R}),
$$

где ұ - нечетное решение краевой задачи (4.3), (1.11) с одним нулем, а постоянная $C$ зависит лииь от $\psi_{0}$ (см. рис. 3 ).

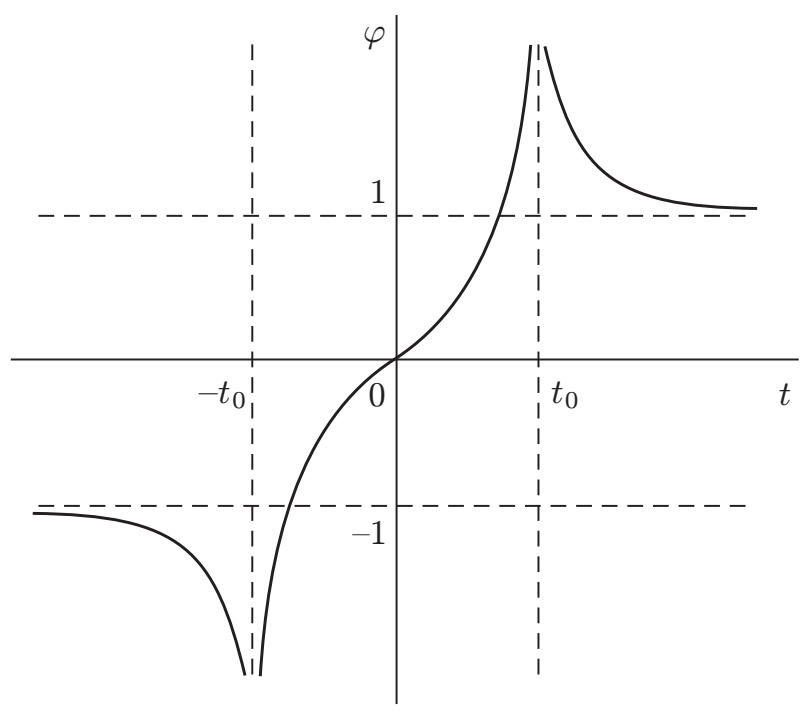

Рис. 3

ЗАмечАниЕ. В качестве решения $\psi_{0}$ в теореме 2 можно взять любое четное решение краевой задачи (1.8a), (1.10), описанное в разделе 3.

В случае $p$ вида $p=4 n+3$ доказательство сходимости изложенного метода не проходит, однако последовательные приближения (4.9), по-видимому, сходятся.

\section{5. СВОЙСТВА ИНТЕГРАЛЬНОГО ОПЕРАТОРА $K_{\gamma}$}

Интегральный оператор $K_{\gamma}$ определяется формулой

$$
\left(K_{\gamma} f\right)(t)=\sqrt{\frac{\gamma}{\pi}} \int_{-\infty}^{\infty} f(\tau) e^{-\gamma(t-\tau)^{2}} d \tau, \quad \gamma>0
$$

на классе $\mathfrak{B}_{\gamma}$ локально интегрируемых функций $f$, удовлетворяющих условию роста

$$
|f(t)|=O\left(e^{\varepsilon t^{2}}\right), \quad|t| \rightarrow \infty, \quad 0 \leqslant \varepsilon<\gamma .
$$


Нам понадобится шкала весовых сепарабельных гильбертовых пространств $\mathfrak{L}_{2}^{\alpha}$, $\alpha>0$, состоящих из измеримых квадратично-суммируемых функций на $\mathbb{R}$ по мере

$$
d \mu_{\alpha}(t)=\sqrt{\frac{\alpha}{\pi}} e^{-\alpha t^{2}} d t, \quad \int_{-\infty}^{\infty} d \mu_{\alpha}(t)=1,
$$

со скалярным произведением и нормой

$$
(f, g)_{\alpha}=\int_{-\infty}^{\infty} f(t) \bar{g}(t) d \mu_{\alpha}(t), \quad\|f\|_{\alpha}=\sqrt{(f, f)_{\alpha}}, \quad f, g \in \mathfrak{L}_{2}^{\alpha} .
$$

Лемма 1. Onepamop $K_{\gamma}$ отображает $\mathfrak{L}_{2}^{\alpha}$ в $\mathfrak{L}_{2}^{\beta}$ npu

$$
0<\alpha<2 \gamma, \quad \beta>\frac{2 \alpha \gamma}{2 \gamma-\alpha}
$$

и ограничен,

$$
\|K f\|_{\beta} \leqslant\left(\frac{\beta \gamma^{2}}{2 \alpha \beta \gamma-2 \alpha^{2} \gamma-\beta \alpha^{2}}\right)^{-1 / 4}\|f\|_{\alpha}, \quad f \in L_{2}^{\alpha} .
$$

Лемма 2. Оператор $K_{\gamma}$ отображает $\mathfrak{L}_{2}^{\alpha}, 0<\alpha<2 \gamma$, в целую функиию $(K f)(z)$, $z=t+i y$, не выше второго порядка роста с оценкой

$$
|(K f)(z)| \leqslant\|f\|_{\alpha} \sqrt{\gamma}(2 \gamma-\alpha)^{-1 / 4} \exp \left(y^{2}-\gamma t^{2}+\frac{2}{2 \gamma-\alpha} t^{2}\right) .
$$

Лемма 3. Оператор $K_{\gamma}$ отображает ограниченную функцию $|f(t)|<C$ в ограниченную функиию $\left|\left(K_{\gamma} f\right)(t)\right|<C$, и если $f(t) \rightarrow$ a при $t \rightarrow \infty$, mо $\left(K_{\gamma} f\right)(t) \rightarrow a$ nрu $t \rightarrow \infty$.

ЛЕмма 4. Если $f, f^{\prime} \in \mathfrak{B}_{\gamma} u f(t)$ - нечетная (четная) непрерывная возрастающая при $t \geqslant 0$ функция, непрерывно дифференцируемая всюду, кроме счетного числа изолированных точек, в окрестности которых $f^{\prime}(t)$ интегрируема, то вещественно-аналитическая функиия $\left(K_{\gamma} f\right)(t)$ нечетная (четная) и возрастает npu $t \geqslant 0$.

ДокАЗАТЕЛЬСтво ЛЕмм. При $\gamma=1$ леммы 1, 2 доказаны в [11], а лемма 3 - в [4]. При $\gamma \neq 1$ их доказательство аналогично. Докажем возрастание функции $\left(K_{\gamma} f\right)(t)$ при $t \geqslant 0$. По предположению функция $f(t)$ не содержит сингулярной части, и поэтому производная $f^{\prime}(t) \geqslant 0$ почти всюду, и справедлива формула интегрирования по частям для произведения с гладкой функцией. Воспользовавшись правилом дифференцирования свертки [3], получим для нечетных $f$ при $t \geqslant 0$ неравенство

$$
\left(K_{\gamma} f\right)^{\prime}(t)=\sqrt{\frac{\gamma}{\pi}} \int_{0}^{\infty} f^{\prime}(\tau)\left[e^{-\gamma(t-\tau)^{2}}+e^{-\gamma(t+\tau)^{2}}\right] d \tau \geqslant 0 .
$$

Аналогичное рассуждение можно провести и для четных $f$. 
Благодарности. Автор сердечно благодарит И. Я. Арефьеву за полезные обсуждения и за численные расчеты. Работа выполнена при частичной финансовой поддержке Программы поддержки ведущих научных школ (грант № НШ1542.2003.1).

\section{Список литературы}

[1] L. Brekke, P. G. O.Freund, Phys. Rep. (Rev. Set. Phys. Lett.), 233:1 (1993), 1.

[2] N. Moeller, M. Schnabl, JHEP, 01 (2004), 011.

[3] И. М. Гельфанд, Г. Е. Шилов, Обобщенные функиии. Вып. 2. Пространства основных и обобщенных функиий, Физматлит, М., 1958.

[4] В. С. Владимиров, Я. И. Волович, ТМФ, 138:3 (2004), 355; math-ph/0306018.

[5] М. Грин, Дж. Шварц, Э. Виттен, Теория суперструн, т. I, II, Мир, М., 1990.

[6] L. Brekke, P. G. O. Freund, M. Olson, E. Witten, Nucl. Phys. B, 302 (1988), 365; В. С. Владимиров, И. В. Волович, Е. И. Зеленов, $p$-Адический анализ и математическал физика, Наука, M., 1994; A. Sen, JHEP, 04 (2002), 048; hep-th/0203211; D. Ghoshal, A. Sen, Nucl. Phys. B, 584 (2000), 300; I. V. Volovich, Class. Quant. Grav., 4 (1987), L83; J. A. Minahan, JHEP, 03 (2001), 028; N. Barnaby, JHEP, 07 (2004), 025; hep-th/0406120; E. Coletti, I. Sigalov, W. Taylor, JHEP, 08 (2005), 104; hep-th/0505031.

[7] P. H. Frampton, Y. Okada, Phys. Rev. D, 37:10 (1988), 3077.

[8] N. Moeller, B. Zwiebach, JHEP, 10 (2002), 034; hep-th/0207107.

[9] I. Ya. Aref'eva, L. V. Joukovskaja, A. S. Koshelev, JHEP, 09 (2003), 012; hep-th/0301137.

[10] Ya. I. Volovich, J. Phys. A, 36 (2003), 8685; math-ph/0301028.

[11] В. С. Владимиров, Изв. РАН. Сер. матем., 69:3 (2005), 55; math-ph/0507018.

[12] Л. В. Жуковская, ТМФ, 146:3 (2006), 402.

[13] G. Calcagni, JHEP, 05 (2006), 012; hep-th/0512259.

[14] В. С. Владимиров, УМН, 60:6 (2005), 73. 